[Radiocarbon, Vol 22, No. 2, 1980, P 159-165]

\title{
VARIATIONS IN RADIOCARBON PRODUCTION IN THE EARTH'S ATMOSPHERE
}

\author{
SERGE A KORFF and ROSALIND B MENDELL \\ Department of Physics, New York University, \\ 4 Washington Place, New York, New York 10003
}

\begin{abstract}
We have investigated solar phenomena associated with unusual changes in the production rates of ${ }^{14} \mathrm{C}$ in the atmosphere. ${ }^{14} \mathrm{C}$ is produced in interactions of cosmic ray neutrons with nitrogen in the atmosphere. Intensity of the neutrons varies globally and fluctuates with time as a result of interactions of galactic cosmic rays which generate neutrons with plasma and magnetic fields of the solar wind. We estimate the total mean production rate of ${ }^{14} \mathrm{C}$ for solar cycle 20 , specifically 1965 to 1975 , to be $2.25 \pm 0.1$ nuclei- $\mathrm{cm}^{-2} \mathrm{sec}^{-1}$ from galactic cosmic rays alone, with negligible integrated contribution from solar particle events. Annual averages of $R_{Z}$, the Zurich sunspot number, and the production rate of ${ }^{14} \mathrm{C}, n\left({ }^{14} \mathrm{C}\right)$, were related by $\mathrm{n}\left({ }^{14} \mathrm{C}\right)=$ $2.60-5.53 \times 10^{-3} \mathrm{R}_{Z} \pm 3$ percent. The contribution of solar flare particles and the zero sunspot limit are discussed with relation to major fluctuations that appear in the radiocarbon versus dendrochronology over short ( -100 years) integration times.
\end{abstract}

\section{INTRODUCTION}

Radiocarbon is mainly generated in the earth's atmosphere by neutrons associated with cosmic radiation. These neutrons are generated by cosmic radiation primaries or energetic secondaries impacting upon the nuclei of atmospheric gases, of which nitrogen constitutes approximately 80 percent. The neutrons are "fast" when generated. They may be captured immediately, or first slowed by collisions with atmospheric constituents. The majority of neutrons will be captured by nitrogen nuclei, most of which will ultimately generate radiocarbon.

Primary cosmic radiation is, by definition, that which reaches the earth from beyond the solar system. Because of their short half-life, few neutrons reach us from the sun, and essentially none from outside the solar system. Primary cosmic radiation is modulated by the solar and interplanetary magnetic fields and by the fluctuations in these fields. The sun exudes a flux of particles called the "solar wind", which in turn carries a magnetic field and modulates the primary radiation. Many of the electrodynamic processes involved are poorly understood at present, but the wind and its variations are being regularly monitored and some data have now been accumulated on its behavior over a number of years. Solar wind shows considerable short-term variation, both in average velocity and in density of particles per unit volume.

\section{Solar variations}

The sun is a fairly quiet and constant star, from the point of view of its major emission of visible electromagnetic radiation. On the other hand, the sun also emits ultraviolet, X-rays and charged particles, and in these spectral regions, the sun shows sometimes rapidly varying amounts. Sunspot numbers, which have been logged for two centuries, seem to be a good index of solar activity. Solar flares, prominences and other manifestations of activity, more or less follow this index. Few of the correlations are precise. We should not think of spots or flares as causes of solar variations. For example, there are often more and larger 
flares in the periods just before and after sunspot maximum than there are at maximum itself. We do not have any generally accepted theory of sunspots or flares or of solar activity in general, although many ideas, each with enthusiastic support, have been suggested.

The sun is surrounded by a corona, visible to the unaided eye at times of solar eclipse. The corona, at times, displays long streamers which the eye can follow for several solar diameters. Recently, "holes" have been found in the corona and apparently, particles from the sun emerge into space through these holes. A larger area of the sun is covered by these holes at times of sunspot minimum than at maximum. Much about solar and coronal physics is simply not understood at the present time.

\section{The sun's magnetic field}

That the sun has a magnetic field of its own was inferred over a century ago by observing the shape of the corona, which resembles a slightly modified dipole. Measurements made with the Zeeman effect indicate that the surface field of the sun is of the order of a few gauss or less. Since the magnetic moment of the sun and the field are related by $\mathrm{M}=\mathrm{Hr}^{3}$, where $\mathrm{r}$ is the radius, $\mathrm{M}$ the dipole moment and $\mathrm{H}$ the field, taking the radius of the sun as $6.965 \times 10^{10} \mathrm{~cm}$, the magnetic moment of the sun is $3.378 \times 10^{32}$ gauss cc per gauss of surface field. At a distance of the radius of the earth's orbit, such a moment would generate a field of the order of $10^{-9}$ gauss per gauss of surface field. A field of this magnitude cannot be detected by present-day techniques. On the other hand, this field pervades the vast volume of the solar system. A charged cosmic ray particle entering such a field would follow a trajectory, if it were fully relativistic, given by $\mathrm{HR}=3 \times 10^{-3} \mathrm{E}$, where $\mathrm{R}$ is the radius of the trajectory and $\mathrm{E}$ the energy in electron volts. (For a classical particle the value of $H R=p / e$ where $\mathrm{p} / \mathrm{e}$ is the momentum per unit charge. However, since almost all primary particles are fully or nearly fully relativistic, we can disregard this). For a cosmic ray particle of $10^{9}$ ev at the low end of the cosmic ray spectrum, the radius of the orbit produced by a field of $10^{-9}$ gauss is many astronomical units. Thus, the sun's field, itself, would do very little bending of high energy cosmic ray particles.

Magnetic fields in sunspots are as much as three orders of magnitude greater than that of the sun itself. However, since these fields are high only over the spot that is small in radius compared to the sun, the moment of a spot is much less than that of the sun, and at the distance of the earth's orbit radius, individual spots will not produce any appreciable effect.

\section{Solar wind}

Solar wind is predominantly a flux of protons, with some alpha particles and a few heavier nuclei, just as is the primary cosmic radiation, itself. The wind blows at about $400 \mathrm{~km} / \mathrm{sec}$ with variations of perhaps $100 \mathrm{~km} / \mathrm{sec}$. It carries with it an average field of about 60 microgauss, which is readily measurable. The orbits of the cosmic ray particles experi- 
ence appreciable bending in this field. Fluctuations in the wind and the field manifest themselves as modulation of the cosmic rays arriving at the earth, and, therefore, as changes in the rates of radiocarbon production.

\section{Effects of solar rotation}

As the sun rotates with a period of approximately 27 days for its middle-latitude regions, the radially outflowing wind is dragged around into a spiral. This is commonly called the "garden hose effect", because it resembles a stream of water coming out of a hose which is rotated about a verticle axis. The sun's rotation introduces a 27-day recurrence effect, observed for many years in magnetic phenomena on earth.

Whereas the changes in radiocarbon production show rather large fluctuations that can be correlated with solar activity, with individual flares, and with the 27-day effect, resultant effects in cumulative quantities of radiocarbon are greatly reduced by smoothing over the relatively long (5700-year) half-life of radiocarbon. Even the 11-year sunspot cycle is rendered almost imperceptible by the long half-life. However, if a long period change in solar emission were to take place, the change in the rate of radiocarbon generation might be observable. Smoothing is further abetted by occasional injection of sudden large doses of radiation occurring at times distant from periods of maximum solar activity. Long-term changes in solar emission have been known to occur. Sunspots were reported by Galileo 400 years ago, but systematic records were not kept until a century later. Records indicate that the sun showed almost no spots between $\mathrm{AD} 1630$ and $\mathrm{AD}$ 1710, which we interpret as an index of low solar activity. This is the "Maunder Minimum", named after a British astronomer who called attention to it (Eddy, 1976; Eddy, Gilman, and Trotter, 1976). Fragmentary records also indicate that during this time the corona did not show long streamers at times of eclipse, and that the Aurora Borealis, which is affected by the solar wind, was seldom seen. We do not know how many other such minima in solar activity have occurred. The sun may have had many prolonged periods of quiet during its geologically recent history. These minima seem to be correlated with periods of deteriorating climate, eg, the "little ice age".

A splendid correlation now exists between radiocarbon and dendrodates (Ralph, Michael, and Han, 1973). This curve shows a major departure from a smooth curve in the interval between AD 1600 and 1700. Around the year 1700, the observed points fall below the smooth line, while in the following years they climb above it. In other words, around 1700 , radiocarbon dates are younger than dendro-dates, while at 1750 they trend in the other direction. From about 1300 to 1400 , the observed points lie almost perfectly on a straight line, ie, radiocarbon dates and the dendrodates match very closely. If the radiocarbon dates are more recent than the dendrodates, more than average radiocarbon was generated, presumably as a result of less than average solar activity. The curve of dendro-dates versus radiocarbon dates shows two important characteristics. First, a clear, long-term sine wave of a 9000 -year wave 
length occurs that has been attributed to a long-term trend in the earth's magnetic moment. Second, there are smaller variations in which the dates depart from a smooth average in both directions. Radiocarbon dates fluctuate above and below dendro values.

For long-term corrections, if the earth's magnetic moment increases, geomagnetic cut-off for incoming radiation rises to higher values and total cosmic radiation, and, therefore, radiocarbon production in the atmosphere diminishes, resulting in radiocarbon dates that seem earlier. The overall effect of the 9000 -year wave is, by now, fairly well understood and corrected for.

We turn next to short-term effects, or, eg, the Maunder minimum. In the interval between 800 and $1800 \mathrm{BC}$, radiocarbon was being produced at close to the average rate, and solar activity was more or less normal.

Consider next the substantial fluctuation in the curve that took place about 2000 BC, an important date in the history of Egypt. The Old Kingdom is usually dated about 2500 to 2200 BC. It apparently disintegrated around $2270 \mathrm{BC}$ and was followed by the Middle Kingdom, a period from 2100 to $1700 \mathrm{BC}$, or the 11th to 13th dynasties. According to most historians, the cause of the collapse of the Old Kingdom is not understood. Further, we do not know whether the collapse and the simultaneous departures in the radiocarbon curve from relatively smooth values is simply coincidental. We merely wish to point out that there is a possibility that the bump in the curve may also indicate the climatic fluctuation, that did not necessarily have to occur in Egypt. A change in the average snowfall on the Ruwenzori or in the Ethiopian highlands would affect the level of the Nile without necessarily any climatic effect in Egypt itself.

\section{Calculation of the effect of solar activity on atmospheric radiocarbon}

Table 1 illustrates the effect of solar activity on the production rates of radiocarbon during recent years. The annual means of the Zurich sunspot number $\left(\mathrm{R}_{\mathrm{Z}}\right)$ and the corresponding radiocarbon production rates $n\left({ }^{14} \mathrm{C}\right)$ for each year of solar cycle 20 are listed. The values of $n\left({ }^{14} \mathrm{C}\right)$ are based on a calculation of the nucleonic cascade in the atmosphere (Light and others, 1973). This calculation incorporated recent cross-section data, observations of the primary solar and galactic cosmic ray spectrum, and our observations of atmospheric neutrons during the first seven years of solar cycle 20. This cycle was the median in sunspot activity during the past 20 cycles, or over 200 years. Based on the data in table 1 , the 11-year mean of $\left.\mathrm{n}^{14} \mathrm{C}\right)$ was $2.25 \pm 0.1$ atoms-cm $^{-2}-\mathrm{sec}^{-1}$, and the annual averages of $n\left({ }^{14} \mathrm{C}\right)$ followed the relation $n\left({ }^{14} \mathrm{C}\right)=2.60$ $-5.53 \times 10^{-3} \mathrm{R}_{\mathrm{Z}}$ atoms- $\mathrm{cm}^{-2}$-sec $^{-1}$, with a 3 percent standard deviation in the calculated $\mathrm{n}\left({ }^{14} \mathrm{C}\right)$. Lingenfelter and Ramaty (1970) used slow neutron data in solar cycle 19 , the most active on record, in an earlier calculation. Although we find a greater dependence of radiocarbon production rates on sunspot actvity, the values we calculate all fall within the 20 percent uncertainties given by the above authors. Thus, 


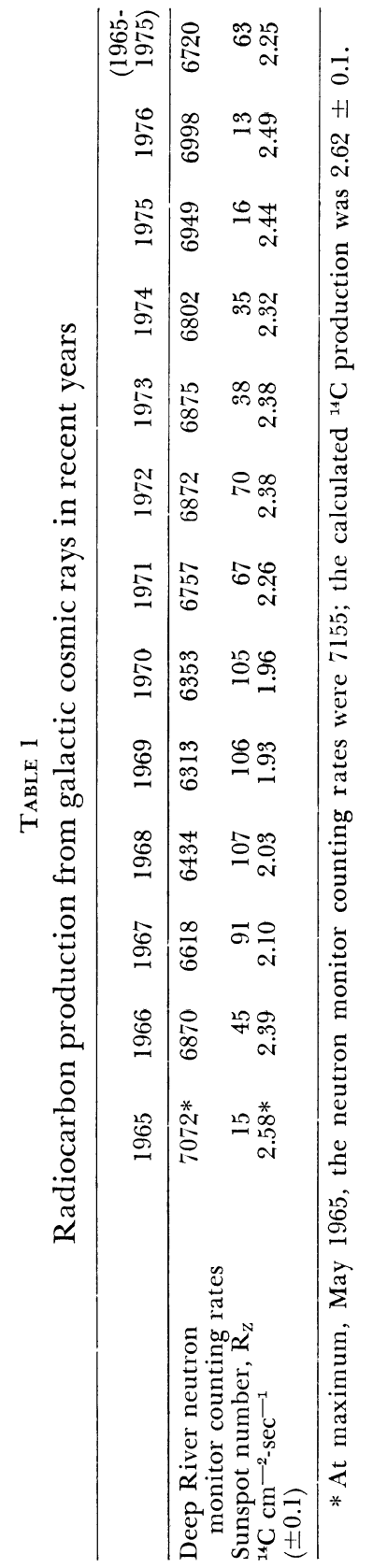


we believe we now have a fair idea of radiocarbon production in average and in very active solar cycles, due to galactic cosmic rays only.

We can also estimate how solar cycle variations affect the radiocarbon budget in the atmosphere. Table 1 shows a peak-to-peak oscillation in $n\left({ }^{14} \mathrm{C}\right)$ of 25 percent. This oscillation is attenuated by a factor of 100 or more (see Damon, 1977), eg, through dilution in the atmosphere and exchange with the biosphere and the deep sea. The net peak-to-peak effect is 0.25 percent or less, which is near the limit of detection. These oscillations are of great interest at present, particularly in determining whether the solar clock that drives the 11-year cycle is a recent phenomenon and whether the sun was more variable in the past than the 11-year phenomenon suggests (Eddy, 1976).

Many investigators believe that the 11-year cycle in radiocarbon dates is also smoothed out by the effect of solar particle emission in solar flares. The explosive flare activity responsible for sweeping galactic cosmic rays out of the solar system can also accelerate solar particles to high energy. The energy spectrum of the solar particle events are steeper than that of galactic cosmic rays, so that there are fewer high energy particles that can penetrate the earth's magnetic field at low latitudes. In general, radiocarbon production from flares is of short duration and is confined to a small fraction of the earth's surface. Our observations of neutrons during solar particle events, reveal that only the most energetic events contribute to the radiocarbon content of the atmosphere (Mendell and others, 1973). In solar cycle 20, the contribution was 0.1 percent. A single flare in solar cycle 19 (rated first in sunspot activity) generated most of an additional 10 percent to radiocarbon from cosmic rays, almost cancelling the difference in production between cycles 19 and 20. If as energetic as this flare, flares in solar cycle 18 (rated third with a sunspot maximum of 150) added 3 percent to the 11-year mean. Thus, radiocarbon excess in the past three cycles decreased exponentially with mean sunspot number. If this represents a trend, we should observe an 11-year wave in the past, with amplitude increasing with sunspot number up to mean sunspot numbers around 70 (11-year mean). At still higher mean sunspot number, the additional loss in ${ }^{14} \mathrm{C}$ production due to the modulation of galactic cosmic rays can have been cancelled and even reversed by the rapidly rising contribution of ${ }^{14} \mathrm{C}$ from high energy solar particles produced in the giant explosive flares. For example, during past periods like the "Grand Maximum" in the 11th to 13th centuries (Eddy, 1976), the decrease in $n\left({ }^{14} \mathrm{C}\right)$, if due to solar activity, would be limited by the above effect.

The Maunder minimum has been attributed to activity characterized by sunspot number 6 to 10 at maximum (Eddy, 1976). If solar cycle 20 gives a fair representation of the relation of sunspot activity to radiocarbon input, the change in the average radiocarbon production was about 2.2 atoms-cm ${ }^{-2}-\mathrm{sec}^{-1}$ in the peak solar cycle of the late 1720 's to about 2.6 when the sunspot number effectively reached 0 . This is about twice the variation usually quoted. Eddy has pointed out that very few 
aurorae were reported during the Maunder minimum and the white light corona was absent from reports of solar eclipses. This describes a period when the sun's dipole field is weak and the solar wind relatively featureless, which could bring solar modulation effects below 1965 levels. A 15 to 20 percent variation in radiocarbon input, suggested by the data, is further attenuated in atmospheric concentration of radiocarbon, depending on the models used for the exchange of radiocarbon in the various reservoirs. The observed deviation of the ${ }^{14} \mathrm{C}$ content from the long-term curve was of the order of $11 / 2$ percent in the intervening following period (Damon, 1977). The predicted attenuation of a 180-year wave is a factor of 10 to 25, the larger values being associated with the more sophisticated models. The estimated increase in ${ }^{14} \mathrm{C}$ production during the Maunder minimum is not inconsistent with the larger factors of attenuation.

\section{ACKNOWLEDGMENT}

This work was supported in part by the National Science Foundation Grant ATM77-107095.

\section{REFERENCES}

Damon, P E, 1977, Solar induced variations of energetic particles at 1 AU, in White, O R, ed, The solar output and its variation: Boulder, Colo., Colorado Assoc Univ Press, p 429-445.

Eddy, J A, 1976, The Maunder Minimum: Science, v 192, p 1189-1201.

Eddy, J A, Gilman, P A, and Trotter, D E, 1976, Solar rotation during the Maunder Minimum: Solar Physics, v 46, p 3-14.

Korff, S A, Mendell, R B, Merker, M, Light, E S, Verschell, H J, and Sandie, W, 1979, Atmospheric neutrons: NASA contractor's rept, 3126, Washington, DC.

Light, E S, Merker, M, Verschell, H J, Mendell, R B, and Korff, S A, 1973, Time dependent worldwide distribution of atmospheric neutrons and their products, 2, Calculation: Jour Geophys Research, v 78, p 2741-2762.

Lingenfelter, R E and Ramaty, R, 1970, Astrophysical and geophysical variations in ${ }^{14} \mathrm{C}$ production, in Olsson, I U, ed, Radiocarbon variations and absolute chronology, Nobel symposium, 12th, Proc: New York, John Wiley and Sons, p 513-537.

Mendell, R B, Verschell, H J, Merker, M, Light, E S, and Korff, S A, 1973, Time dependent worldwide distribution of atmospheric neutrons and their products. 3 . Neutrons from solar protons: Jour Geophys Research, v 78, p 2763-2778.

Ralph, E K, Michael, H N, and Han, M C, 1973, Radiocarbon dates and reality: MASCA Newsletter, v 9, no. 1, p 1-20. 